\title{
Experimental study of the robust global synchronization of Brockett oscillators
}

\author{
Hafiz Ahmed $^{1, a}$, Rosane Ushirobira ${ }^{2}$, and Denis Efimov ${ }^{2}$ \\ 1 Department of Electrical and Computer Engineering, Clemson University, Clemson, SC \\ 29634, USA. \\ 2 Inria, Non-A team, 40 avenue Halley, 59650 Villeneuve d'Ascq, France.
}

\begin{abstract}
This article studies the experimental synchronization of a family of a recently proposed oscillator model, i.e. the Brockett oscillator [Brockett, 2013]. Due to its structural property, Brockett oscillator can be considered as a promising benchmark nonlinear model for investigating synchronization and the consensus phenomena. Our experimental setup consists of analog circuit realizations of a network of Brockett oscillators. Experimental results obtained in this work correspond to the prior theoretical findings.
\end{abstract}

\section{Introduction}

Over the last decades, the synchronization of complex dynamical systems and/or network of systems has attracted a great deal of attention from multidisciplinary research communities thanks to their pervasive presence in nature, technology and human society $[1,2]$. One critical issue arising from complex systems is to develop distributed control policies based on local information that enables all subsystems to reach an agreement on certain quantities of interest, this is known as the consensus problem. A classic example of distributed coordination/consensus in physics, engineering and biology is the synchronization of arrays of coupled nonlinear oscillators $[3,4,5]$. Synchronization of oscillators network has several potential application domains, for example, chaos synchronization $[6,7,8,9]$, smooth operations of microgrid $[10,11]$, secure communication [12], formation control [13], genetic oscillators [14, 15], etc.

Significant progresses have been made during the past decades in the area of synchronization of oscillator network. The existing literature is huge and covers a wide area of topics. Interested readers may consult $[16,17,18]$ to have a global overview. However, the major focus of the existing literature is on the development of analytical and/or numerical results. Much less attention is devoted to the verification of theoretical synchronization results in real experimental setups. In order to apply the existing results to practical problems, more studies on experimental verification are needed. The focus of this article is on the experimental realization of existing theoretical results.

a e-mail: hafiza@clemson.edu 
The existing literature on experimental studies are not as mature as the analytical and/or numerical results but it started to gain the attention of research communities in various scientific disciplines. In [19], through hardware experiments, authors have validated their prior theoretical results on synchronization and partial synchronization of networks of time-delay coupled Hindmarsh-Rose neurons. Theoretical results predicted exact synchronization but in practice the authors were able to achieve practical synchronization (i.e. boundedness of the synchronization error instead of convergence to zero) in [19]. Experimental validation of emergent behavior in networks of Chua's circuits is conducted in [20]. However, the underlying theoretical results are not analytical in [20]. Verification of numerical results on the synchronization of coupled electrical self-oscillators through experiment can be found in [21]. Experiments on the synchronization of synaptically coupled nonlinear oscillators can be found in [22]. Experimental synchronization in the case of ring of periodic oscillators can be found in $[23,24]$.

A good number of experimental verification reported in the literature using the coupling values found in simulations is based on comparison with numerical results. This opens up the scope of experimental verification of analytical results which is often not an easy task (cf. [19]). All this motivates the current work. The objective of this article is to provide experimental synchronization results for a family of Brockett oscillators [25]. A Brockett oscillator can be expressed by the following model in state-space form [26]:

$$
\begin{aligned}
& \dot{x}_{1}=x_{2} \\
& \dot{x}_{2}=-x_{1}+a u+b x_{2}\left(x_{1}^{2}+x_{2}^{2}-1\right), a, b>0
\end{aligned}
$$

Details of the model (1) can be found in the Appendix. The specialty of model (1) is that depending on model parameters, the conventional averaging theory does not predict the existence of a periodic (almost periodic) solution. However, qualitative synchronization together with small amplitude irregular motion can be seen through numerical studies [25]. By qualitative synchronization, we mean here the theoretical prediction about synchronization without any quantitative information i.e. the rate of convergence or the domain of attraction.

In [27], authors have analyzed the global stability and input-to-state stability (ISS) of model (1) with respect to equilibrium at the origin and the limit cycle at the unit sphere. These results make Brockett oscillator a promising benchmark nonlinear model for investigation of synchronization and consensus phenomena. Global synchronization result for a family of Brockett oscillators can also be found in [27]. The summary of the results obtained in [27] can be found in the Appendix. Moreover, in [28] it is shown that it is possible to globally synchronize a family of nonidentical nonlinear systems by transforming individual subsystems into the form of Brockett oscillators (1). So, the experimental verification of results obtained in [27] has a practical interest for readers interested in investigating the synchronization and consensus phenomena in nonlinear systems. This experimental verification is the main contribution of this article.

The rest of the article is organized as follows: analog circuit realization of model (1) can be found in Section 2, numerical simulation results are given in Section 3 while the experimental counterpart can be found in Section 4. Finally, Section 5 concludes this work. A summary of the analytical results obtained in [27] can be found in the Appendix. 


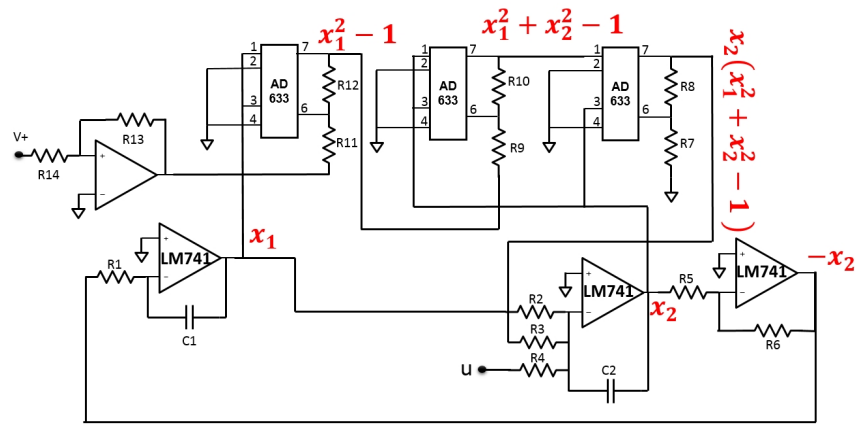

Fig. 1. Schematic of the Brockett oscillator. Parts of eq. (1) are denoted by red terms. Parameters a and b are determined by the resistors $R_{4}$ and $R_{3}$ respectively.

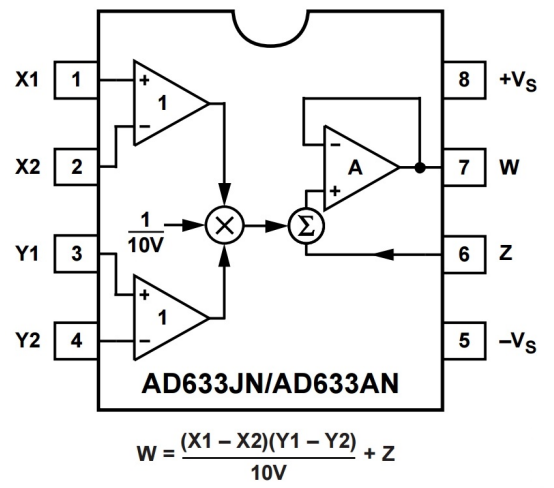

Fig. 2. Pin number of an $\mathrm{AD} 633 \mathrm{JN}$ analog multiplier.

\section{Analog implementation of the Brockett oscillator}

The analog circuit diagram of a Brockett oscillator can be seen in Fig. 1. To implement the multiplication operation of model (1), we have used analog multipliers. The multipliers are Analog Device AD $633 \mathrm{JN}$ (see Fig. 2 for pin numbers) version of the AD 633 four-quadrant voltage multipliers chips. They operate over a dynamic range of $\pm 11 \mathrm{~V}$ with a typical error less than $1 \%$. They also have a built-in divide-by-ten feature. The signal at output pin 7 (W) depends on inputs X1, X2, Y1, Y2 and Z which is the summing input. The output can be expressed by the following equation:

$$
\mathrm{W}=\frac{(\mathrm{X} 1-\mathrm{X} 2)(\mathrm{Y} 1-\mathrm{Y} 2)}{10}+\mathrm{Z}
$$

The integrators are operational amplifiers (LM 741) with feedback capacitors and summations are accomplished by operational amplifiers with multiple input resistors. The values of the various resistors and capacitors of Fig. 1 are: R1, R2, R3, R4 = $100 \mathrm{~K} \Omega ; \mathrm{R} 5, \mathrm{R} 6=1 \mathrm{~K} \Omega ; \mathrm{R} 7, \mathrm{R} 9, \mathrm{R} 11=1.3 \mathrm{~K} \Omega ; \mathrm{R} 8, \mathrm{R} 10, \mathrm{R} 12=170 \Omega ; \mathrm{R} 13=1 K \Omega$; $\mathrm{R} 14=220 \Omega$ and $\mathrm{C} 1, \mathrm{C} 2=100 \mathrm{nF}$. Model parameters are dependent on R3 and R4. With these values of the parameters, the response of the autonomous Brockett oscillator can be seen in Fig. 3. From Fig. 3, sustained oscillation can be seen along with the convergence of the oscillator state into the unit circle in the $\left(x_{1}, x_{2}\right)$-space. The experimental results of Fig. 3 correspond to the mathematical model (1) since 


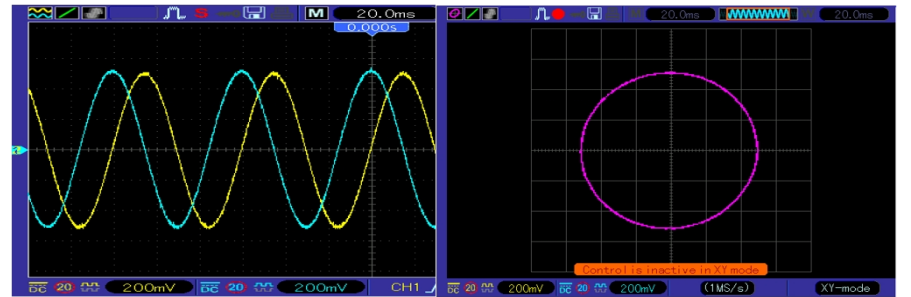

Fig. 3. Experimental implementation results of model (1). Left) Evolution of the state variables - $x_{1}$ (cyan), $x_{2}$ (yellow); and right) $x_{1}$ vs. $x_{2}$ plot.

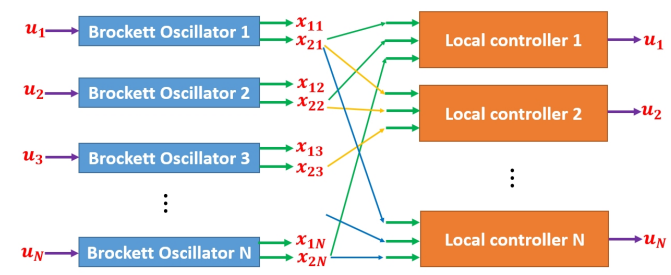

Fig. 4. Simulation setup with control (5) for $N$ Brockett oscillators in an $N$-cycle graph settings.

the trajectories approach the limit cycle on the unit sphere. This demonstrates the sufficient accuracy of the analog implementation.

\section{Numerical simulation results}

In this section, numerical simulation studies will be performed to check the effectiveness of the synchronization control (5) (given in the Appendix). An overview of the simulation setup can be seen in Fig. 4. All the simulations of this section were done using Matlab/Simulink software with Runge-Kutta method and the step size is 0.01. The synchronizing control being used in this work is given as

$$
u=k M\left[\begin{array}{c}
x_{21} \\
\vdots \\
x_{2(N-1)} \\
x_{2 N}
\end{array}\right], M=\left[\begin{array}{ccccc}
-2 & 1 & 0 & \cdots & 1 \\
1 & -2 & 1 & \cdots & 0 \\
0 & 1 & -2 & \cdots & 0 \\
\vdots & & \vdots & \ddots & 0 \\
1 & \cdots & 0 & 1 & -2
\end{array}\right]
$$

where $k>0$ is the coupling strength and $N$ indicates the number of oscillator being used for the simulation. Further details can be found in the Appendix. Two cases will be considered:

\subsection{Case-1: identical oscillators}

Four identical Brockett oscillators are considered for the purpose of synchronization. The model parameters are $k=0.5 a_{i}=0.5, b_{i}=1, i=1, \ldots, 4$. The initial conditions are selected as: $(-2,0),(0,-2),(2,0)$ and $(0,2)$. The model parameters satisfy the condition of Theorem 1 (given in the Appendix). As a result, oscillators are supposed to converge to the set $\Omega_{\infty}^{\prime}$. The result of the simulation in this case can be seen in Fig. 5. 


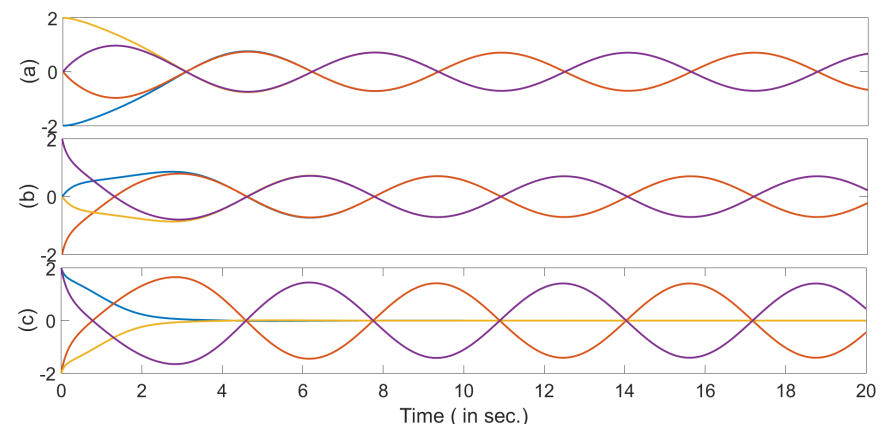

Fig. 5. Simulation results for 4 identical oscillators (a) $x_{1 i}$ ( $x_{11^{-}}$cyan, $x_{12^{-}}$dark magenta, $x_{13}$ - yellow, $x_{14}$ - purple) (b) $x_{2 i}$ ( $x_{21}$ - cyan, $x_{22}$ - dark magenta, $x_{23^{-}}$yellow, $x_{24}$ - purple) (c) $e_{2 i}=x_{2 i}-x_{2(i+1)},\left(e_{2}\right.$ - cyan, $e_{4}$ - dark magenta, $e_{6^{-}}$yellow, $e_{8^{-}}$purple $) i=1, \ldots, 4$

From Fig. 5, anti-phase synchronization can be observed. As a result, some errors converge to zero while others didn't (Fig. 5c). The oscillators converge to a circle which is in $\Omega_{\infty}^{\prime}$ and that corresponds to the theoretical prediction of Theorem 1 (given in the Appendix). In this case, the radius of the circle depends on the system parameters and initial conditions. By changing the parameters and initial conditions, an in-phase synchronization may be observed for identical oscillators. The possible relations of phases and amplitudes of oscillation among the neighboring agents are given by equations (6) and (7) (given in the Appendix). These equations may have more than one solutions. As such various synchronization patterns are possible. Finding some analytical conditions that predict systemic transition from "anti-phase" to "in-phase" synchronization can be considered as an extension to this work in the future.

\subsection{Case-2: nonidentical oscillators}

From a practical point of view, it is often very difficult to realize a family of identical oscillators. As a result, frequently practical systems are non-identical. The results of [27] work for both identical and nonidentical oscillators. In this section, we will consider the synchronization of 4 nonidentical oscillators. The parameters of the oscillators are: $k=1, a_{1}=1, a_{2}=2, a_{3}, a_{4}=3, b_{1}=5, b_{2}=6, b_{3}=7, b_{4}=8$. The initial conditions are same as case- 1 . The model parameters in this case satisfy the condition of Theorem 1 (given in the Appendix).

In this case, an in-phase synchronization can be observed from Fig. 6 . As a result, the errors converge to zero (Fig. 6c). The oscillators converge to the unit circle which is inside the set $\Omega_{\infty}^{\prime}$.

In case-1, an anti-phase synchronization is observed. Adding a small perturbation in the case of identical oscillators leads to in-phase synchronization (difference in parameters for non-identical oscillators can be considered as a perturbation also). Thus, in this example, synchronization is a phenomenon that follows the agents imperfections rather than similarity.

Further simulation have been done to check the effect of initial conditions on synchronization for the case of non-identical oscillators. In this case, we have run the simulation for 100 times with randomly distributed initial conditions within the range $[-2,2]$. The error among the state variables can be seen in Fig. 7 and the histogram 


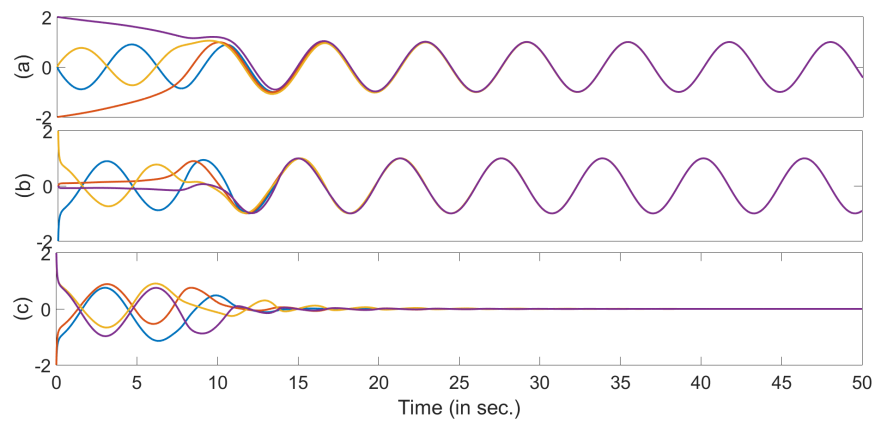

Fig. 6. Simulation results for 4 nonidentical oscillators (a) $x_{1 i}$ (b) $x_{2 i}$ (c) $e_{2 i}=x_{2 i}-x_{2(i+1)}$, $i=1, \ldots, 4$. Color codes are similar to that of Fig. 5 .

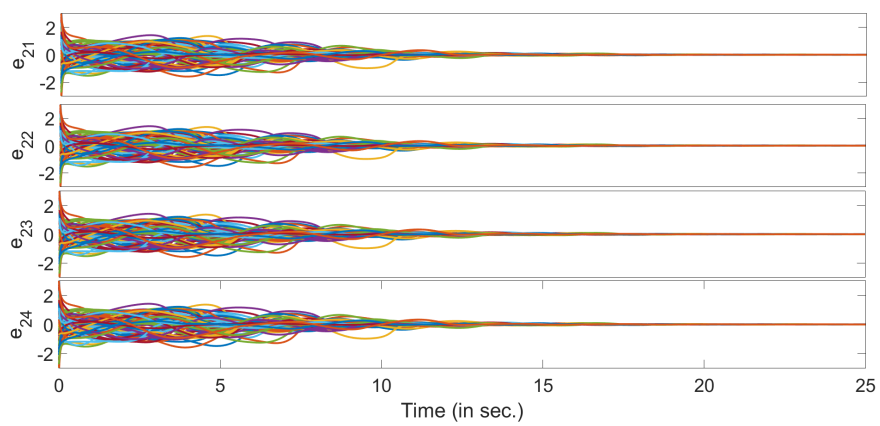

Fig. 7. Evolution of errors $e_{2 i}=x_{2 i}-x_{2(i+1}, i=1, \ldots, 4$ for the case of nonidentical oscillators with randomly selected initial conditions

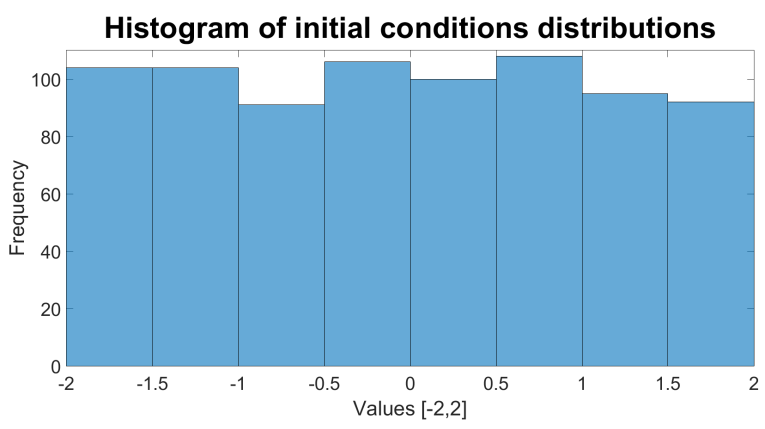

Fig. 8. Histogram of initial conditions distributions.

of the initial conditions distribution can be seen in Fig. $8^{1}$. From Fig. 7, in-phase synchronization can be observed among the oscillators with randomly distributed initial conditions. This illustrates the independence of the synchronization w.r.t initial conditions variation.

\footnotetext{
1 Four oscillators are used for the simulation. Each of them has 2 initial conditions. In each simulation run, different initial conditions were used for all oscillators. So, eight different initial condition points are needed for each simulation run. To run the simulation 100 times, 800 points as initial conditions are needed to be selected. That is why the sum of the bars in Fig. 8 is 800 .
} 


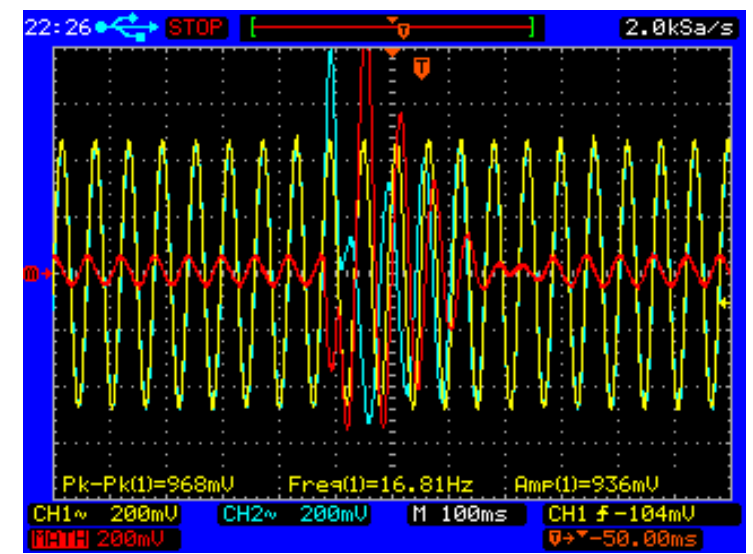

Fig. 9. Synchronization and re-synchronization after perturbation with low control gain. $x_{21}$ (yellow), $x_{22}$ (cyan) and $x_{21}-x_{22}$ (red).

\section{Experimental synchronization results}

This section is devoted to the demonstration of experimental synchronization results. Due to experimental complexities, we have considered $N=3$ oscillators in this section which is the minimum number of oscillators that are needed to implement control (5). For the selection of parameters, we have selected identical values for the family. However, nonidealities and nonlinearities of the practical devices make the oscillators nonidentical in a practical settings. For example, the resistors that are used in this work have a tolerance limit of $\pm 10 \%$. So, even if we take the resistors of same nominal values for individual oscillators, the final values are different due to the tolerance limit. Moreover, each oscillator was powered with a separate power supply. In this work, we have used RB-0512D RECOM power isolated DC/DC converters to generate the biasing voltages for the op-amps. This also introduces some perturbation in the family. In this section, two experimental conditions will be considered.

In the first case, low control gain is selected as in Section 3. The response of oscillators 1 and 2 in this case can be seen in Fig. 9. From the theoretical results, oscillators are supposed to be synchronized with zero error. However, experimental results show that the oscillators are synchronized but the error is not zero. The nonzero synchronization error is due to the imperfection, nonlinearities and nonidealities of the analog implementation. Moreover, this result also shows the limitation of numerical simulations. To have a better picture of the synchronization mismatch, Fig. 10 can be seen. From Fig. 10a, it can be concluded that very little phase difference exists between oscillators 1 and 2. Moreover, in the case of perfect synchronization, $x_{21}$ vs $x_{22}$ graph is supposed to be a straight line. However, Fig. 9b demonstrates that it has an ellipsoidal form instead of a straight line.

To overcome the aforementioned problems, one way is to use a high control gain (i.e. using a high $k$ ). The model parameters are tuned in a way such that the condition of Theorem 1 (given in the Appendix) is always satisfied. The response of oscillator 1 and 2 with high control gain case can be seen in Fig. 11. Due to high control gain, in this case we can see perfect synchronization with negligible error. In a practical implementation, it is not possible to have perfect zero error due to practical constraints like measurement noise, discretization of the continuous signals by the digital oscilloscope etc.. However, if the error is very close to zero, then it can be considered as zero in a practical setup. In Fig. $12 \mathrm{~b}$, a perfect straight line can be seen instead of ellipsoidal shape which shows perfect phase matching of the oscillators. Moreover, in 


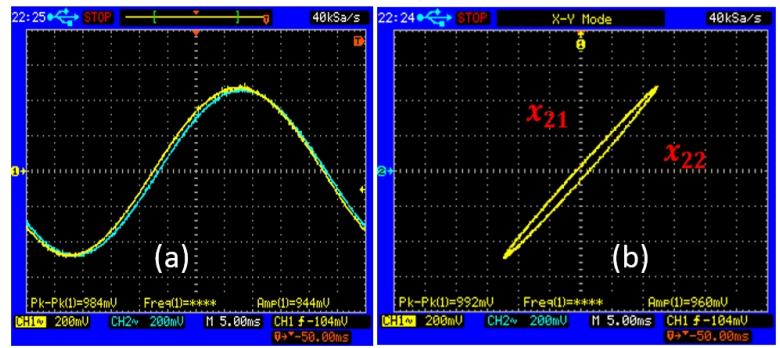

Fig. 10. Low control gain case (a) Superimposed plot of $x_{21}$ and $x_{22}$ and (b) $x_{21}$ vs $x_{22}$ plot

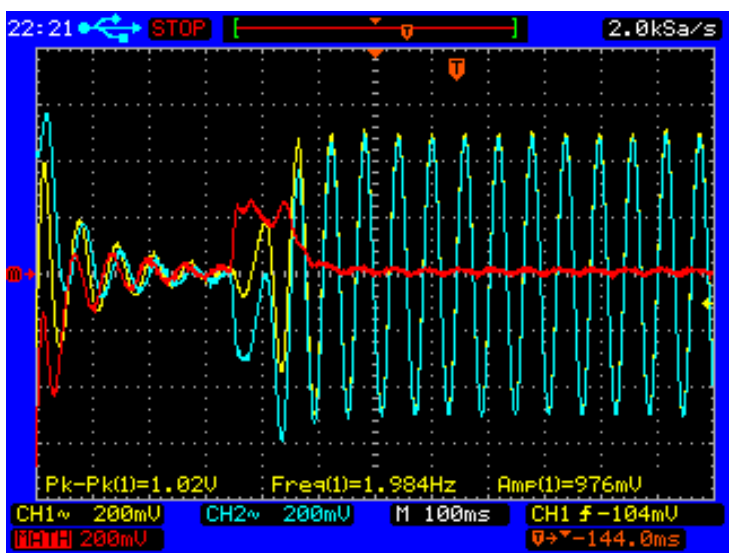

Fig. 11. Synchronization and re-synchronization after perturbation with high control gain. $x_{21}$ (yellow), $x_{22}$ (cyan) and $x_{21}-x_{22}$ (red).

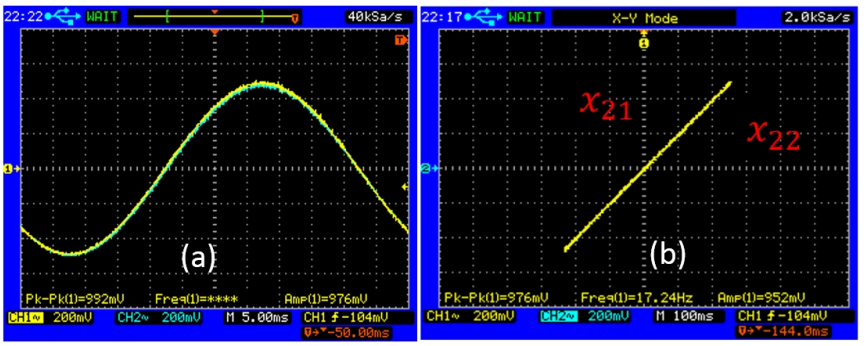

Fig. 12. High control gain case: (a) Superimposed plot of $x_{21}$ and $x_{22}$ and (b) $x_{21}$ vs $x_{22}$ plot

Fig. 12a, the signals are superimposed on each other and no difference can be seen. Also, the results in this case coincide with theoretical findings.

The results of [26] provide robust synchronization for $N=2$ Brockett oscillators. When $N>2$, theoretical results of [27] provide asymptotic synchronization not the robust one. However, through the experimental results, we have seen that the synchronization is quite robust to perturbations. For example in Fig. 9, oscillators are initially synchronized. Then a perturbation is added and the synchronization is lost. As soon as the perturbation disappeared, oscillators returned back to synchronous state. Similar phenomena can be seen in Fig. 11 also. Moreover, it was observed during further laboratory experiments (not reported here) that the synchronization is not lost in the case of small amplitude persistent perturbations. All these facts 
demonstrate experimentally the effectiveness of the synchronization control (5) to synchronize a network of non-identical Brockett oscillators.

\section{Conclusion}

We have studied synchronization in a network of Brockett oscillators that are coupled through error feedback. The theoretical results of [27] are tested using an experimental setup built around electronic circuit realizations of networks of Brockett oscillators. Both numerical and experimental results are provided. Experimental results showed the limitation of numerical simulations. Experimental results obtained in this work correspond to the theoretical findings.

In [27], asymptotic synchronization result was obtained when the number of oscillators are greater than 2. However, it is found through hardware experiments in this work that the synchronization protocol of [27] is robust also. In future, obtaining analytical results on robust synchronization for $N>2$ Brockett oscillators can be considered as an interesting direction of research. Moreover, in this work, an $N$ cycle graph was considered. Other kind of network topology can be explored in future for experimental studies. Finally, the development of a modular framework for the experimental study of synchronization of oscillator network can be considered as an extension to this work. The proposed platform can be used in education process since the oscillator model is sufficiently simple, yet demonstrating a rich oscillatory behavior and admitting an analytical analysis.

The work is partly supported by ANR project WaQMoS (ANR 15 CE 04 0002).

\section{References}

1. S. H. Strogatz, Sync: How order emerges from chaos in the universe, nature, and daily life. Hyperion, 2003.

2. H. Ahmed, R. Ushirobira, D. Efimov, and W. Perruquetti, "Robust synchronization for multistable systems," IEEE Transactions on Automatic Control, vol. 61, no. 6, pp. 1625-1630, 2016.

3. R. Sepulchre, D. Paley, and N. Leonard, "Collective motion and oscillator synchronization," in Cooperative control, pp. 189-205, Springer, 2005.

4. F. Dörfler, M. Chertkov, and F. Bullo, "Synchronization in complex oscillator networks and smart grids," Proceedings of the National Academy of Sciences, vol. 110, no. 6, pp. 2005-2010, 2013.

5. T. Bountis, "From mechanical to biological oscillator networks: The role of long range interactions," The European Physical Journal Special Topics, vol. 225, no. 67, pp. 1017-1035, 2016.

6. A. Loría, "Master-slave synchronization of 4th order Lü chaotic oscillators via linear output feedback," IEEE Transactions on Circuits and Systems II: Express Briefs, vol. 57, no. 3, pp. 213-217, 2010.

7. A. Loría, "A linear time-varying controller for synchronization of Lü chaotic systems with one input," IEEE Transactions on Circuits and Systems II: Express Briefs, vol. 56, no. 8, pp. 674-678, 2009.

8. A. Rodriguez, J. De Leon, and L. Fridman, "Synchronization in reduced-order of chaotic systems via control approaches based on high-order sliding-mode observer," Chaos, Solitons $\&$ Fractals, vol. 42, no. 5, pp. 3219-3233, 2009. 
9. A. Rodríguez, J. De León, and L. Fridman, "Quasi-continuous high-order slidingmode controllers for reduced-order chaos synchronization," International Journal of Non-Linear Mechanics, vol. 43, no. 9, pp. 948-961, 2008.

10. D. Efimov, J. Schiffer, and R. Ortega, "Robustness of delayed multistable systems with application to droop-controlled inverter-based microgrids," International Journal of Control, vol. 89, no. 5, pp. 909-918, 2016.

11. J. Schiffer, T. Seel, J. Raisch, and T. Sezi, "A consensus-based distributed voltage control for reactive power sharing in microgrids," in Control Conference (ECC), 2014 European, pp. 1299-1305, IEEE, 2014.

12. A. L. Fradkov and A. Y. Markov, "Adaptive synchronization of chaotic systems based on speed gradient method and passification," IEEE Transactions on Circuits and Systems I: Fundamental Theory and Applications, vol. 44, no. 10, pp. 905-912, 1997.

13. B. Andrievsky, S. Tomashevich, A. L. Fradkov, and K. Amelin, "Quadrocopters formation control over the limited-band communication network," IFACPapersOnLine, vol. 48, no. 9, pp. 85-90, 2015.

14. H. Ahmed, R. Ushirobira, and D. Efimov, "On robustness of phase resetting to cell division under entrainment," Journal of theoretical biology, vol. 387, pp. 206$213,2015$.

15. D. Efimov, "Phase resetting for a network of oscillators via phase response curve approach," Biological cybernetics, vol. 109, no. 1, pp. 95-108, 2015.

16. R. Olfati-Saber and R. M. Murray, "Consensus problems in networks of agents with switching topology and time-delays," IEEE Transactions on automatic control, vol. 49, no. 9, pp. 1520-1533, 2004.

17. R. Olfati-Saber, J. A. Fax, and R. M. Murray, "Consensus and cooperation in networked multi-agent systems," Proceedings of the IEEE, vol. 95, no. 1, pp. 215233, 2007.

18. E. Panteley and A. Loría, "Synchronisation and emergent behaviour in networks of heterogeneous systems: A control theory perspective," in Nonlinear Systems, pp. 81-102, Springer, 2017.

19. E. Steur, C. Murguia, R. H. Fey, and H. Nijmeijer, "Synchronization and partial synchronization experiments with networks of time-delay coupled HindmarshRose neurons," International Journal of Bifurcation and Chaos, vol. 26, no. 07, p. 1650111, 2016.

20. M. Magistris, M. Bernardo, S. Manfredi, C. Petrarca, and S. Yaghouti, "Modular experimental setup for real-time analysis of emergent behavior in networks of chua's circuits," International Journal of Circuit Theory and Applications, 2015.

21. L. Q. English, Z. Zeng, and D. Mertens, "Experimental study of synchronization of coupled electrical self-oscillators and comparison to the sakaguchi-kuramoto model," Physical Review E, vol. 92, no. 5, p. 052912, 2015.

22. D. Saha, P. Saha, A. Ray, and A. Roychowdhury, "On the synchronization of synaptically coupled nonlinear oscillators: Theory and experiment," Annual Review of Chaos Theory, Bifurcations and Dynamical Systems, p. 1, 2016.

23. C. R. Williams, F. Sorrentino, T. E. Murphy, and R. Roy, "Synchronization states and multistability in a ring of periodic oscillators: Experimentally variable coupling delays," Chaos: An Interdisciplinary Journal of Nonlinear Science, vol. 23, no. 4, p. 043117, 2013.

24. C. R. Williams, T. E. Murphy, R. Roy, F. Sorrentino, T. Dahms, and E. Schöll, "Experimental observations of group synchrony in a system of chaotic optoelectronic oscillators," Physical review letters, vol. 110, no. 6, p. 064104, 2013.

25. R. Brockett, "Synchronization without periodicity," 2013.

26. H. Ahmed, R. Ushirobira, and D. Efimov, "On the robust synchronization of brockett oscillators," IFAC-PapersOnLine, vol. 49, no. 14, pp. 142-147, 2016. 
27. H. Ahmed, R. Ushirobira, and D. Efimov, "Robust global synchronization of brockett oscillators," Technical report, Inria Lille - Nord Europe, Nov. 2016 (available online at https://hal.inria.fr/hal-01391120).

28. H. Ahmed, R. Ushirobira, D. Efimov, and L. Fridman, "Oscillatory global output synchronization of nonidentical nonlinear systems," IFAC World Congress, Toulouse, France, 2017.

\section{Appendix: Synchronization of Brockett oscillators [27]}

The following family of Brockett oscillators [27] is considered for some $N>1$ :

$$
\begin{aligned}
& \dot{x}_{1 i}=x_{2 i}, \\
& \dot{x}_{2 i}=a_{i} u_{i}-x_{1 i}-b_{i} x_{2 i}\left(\left|x_{i}\right|^{2}-1\right), i=1, \ldots, N,
\end{aligned}
$$

where $a_{i}, b_{i}>0$ are the parameters of an individual oscillator, the state $x_{i}=$ $\left[\begin{array}{ll}x_{1 i} & x_{2 i}\end{array}\right]^{T} \in \mathbb{R}^{2}$, the control $u_{i} \in \mathbb{R}\left(u_{i}: \mathbb{R}_{+} \rightarrow \mathbb{R}\right.$ is locally essentially bounded and measurable signal). Denote the common state vector of (4) as $x=\left[x_{1}^{T}, \ldots, x_{N}^{T}\right]^{T} \in$ $\mathbb{R}^{2 N}$ and $u=\left[u_{1}, \ldots, u_{N}\right]^{T} \in \mathbb{R}^{N}$ is the common input.

The following synchronizing control is selected for family (4),

$$
u=k M\left[\begin{array}{c}
x_{21} \\
\vdots \\
x_{2(N-1)} \\
x_{2 N}
\end{array}\right], M=\left[\begin{array}{ccccc}
-2 & 1 & 0 & \cdots & 1 \\
1 & -2 & 1 & \cdots & 0 \\
0 & 1 & -2 & \cdots & 0 \\
\vdots & & \vdots & \ddots & 0 \\
1 & \cdots & 0 & 1 & -2
\end{array}\right]
$$

where $k>0$ is the coupling strength and then from a graph theory point of view, the oscillators are connected through a $N$-cycle graph (each oscillator needs only the information of its left and right neighbor). Next, let us define the synchronization error among the various states of the oscillators:

$$
e_{2 i-1}=x_{1 i}-x_{1(i+1)}, \dot{e}_{2 i-1}=x_{2 i}-x_{2(i+1)}=e_{2 i}
$$

and $e_{2 N-1}=x_{1 N}-x_{11}, \dot{e}_{2 N-1}=x_{2 N}-x_{21}=e_{2 N}$. Then the main results of [27] can be summarized as below:

Proposition 1 For any $k>0$ in the system (4), (5) all trajectories are bounded and converge to the largest invariant set in

$$
\begin{aligned}
\Omega_{\infty}=\left\{x \in M:\left|x_{i}\right|\right. & =\text { const }, e_{2 i-1}^{2}+e_{2 i}^{2}=\text { const } \\
x_{2(i-1)}+x_{2(i+1)} & \left.=\left(2+\frac{b_{i}}{a_{i} k}\left(\left|x_{i}\right|^{2}-1\right)\right) x_{2 i}, i=1, \ldots, N\right\} .
\end{aligned}
$$

Theorem 1 For any $k>0$, if there is an index $1 \leq i \leq N$ such that $2 a_{i} k<b_{i}$, then in the system (4), (5) all trajectories are bounded and almost all of them converge to the largest invariant set in

$$
\begin{aligned}
\Omega_{\infty}^{\prime}=\left\{x \in M:\left|x_{i}\right|\right. & =\text { const } \neq 0, e_{2 i-1}^{2}+e_{2 i}^{2}=\text { const } \\
x_{2(i-1)}+x_{2(i+1)} & \left.=\left(2+\frac{b_{i}}{a_{i} k}\left(\left|x_{i}\right|^{2}-1\right)\right) x_{2 i}, i=1, \ldots, N\right\} .
\end{aligned}
$$


On the set $\Omega_{\infty}^{\prime}$ we have $x_{2 i}=r_{i} \sin \left(t+\phi_{i}\right)$ for all $i=1, \ldots, N$, where $r_{i}=$ $\sqrt{x_{1 i}^{2}+x_{2 i}^{2}}$ and $\phi_{i} \in[0,2 \pi)$ are some constants depending on the system parameters and initial conditions, and

$$
\begin{gathered}
\left(2+\alpha_{i}\left(r_{i}^{2}-1\right)\right) r_{i}=\sqrt{r_{i-1}^{2}+r_{i+1}^{2}+2 r_{i-1} r_{i+1} \cos \left(\phi_{i+1}-\phi_{i-1}\right)}, \\
\phi_{i}=\phi_{i-1}+\arctan \left(\frac{r_{i+1} \sin \left(\phi_{i+1}-\phi_{i-1}\right)}{r_{i-1}+r_{i+1} \cos \left(\phi_{i+1}-\phi_{i-1}\right)}\right)
\end{gathered}
$$

providing expressions of possible relations of phases and amplitudes of oscillation for neighboring agents in (4), (5). 